\title{
THE OSCILLATORY BEHAVIOR OF CERTAIN DERIVATIVES
}

BY

RICHARD J. O'MALLEY AND CLIFFORD E. WEIL

This paper is dedicated to Casper Goffman

\begin{abstract}
The derivatives considered are the approximate derivative and the $k$ th Peano derivative. The main results strengthen the Darboux property, which both of these derivatives possess. THEOREM. If the approximate derivative $f_{\text {ap }}^{\prime}$ of $f$ exists on an interval and if, for $M>0, f_{\text {ap }}^{\prime}$ attains both $M$ and $-M$, then there is an open subinterval where $f_{\text {ap }}^{\prime}=f^{\prime}$ and on which $f^{\prime}$ attains both $M$ and $-M$. The other main theorem is obtained from this one by replacing the approximate derivative by the $k$ th Peano derivative.
\end{abstract}

1. Introduction. The oscillatory behavior of approximate derivatives and $k$ th Peano derivatives is the main topic of this paper. The major problem to be solved is common to both types of derivatives, but separate proofs must be given for each type. In this section, however, the problem is discussed only for the approximate derivative.

Suppose a function $f$ has an approximate derivative $f_{\text {ap }}^{\prime}(x)$ at each $x$ in a fixed interval $I_{0}$. Essential to the development is the following theorem which can be found in [1] or [6] for the approximate derivative and in [4] or [7] for the $k$ th Peano derivative.

THEOREM A. If $f_{\mathrm{ap}}^{\prime}$ exists everywhere on $[a, b]$ and is bounded above or below on $(a, b)$, then $f_{\mathrm{ap}}^{\prime}=f^{\prime}$ on $[a, b]$ (one-sided at $a$ and $b$ ).

Since the approximate derivative possesses the Darboux property, Theorem A forces $f_{\text {ap }}^{\prime}$ to attain every value indeed infinitely of ten on any interval where $f_{\text {ap }}^{\prime}$ is not $f^{\prime}$. Thus $f_{\text {ap }}^{\prime}$ must oscillate between positive and negative values whose absolute value may be as large as desired.

On the other hand, since the approximate derivative is a function of Baire class one, Theorem A implies the existence of an open, dense subset $O$ of $I_{0}$ on which $f_{\mathrm{ap}}^{\prime}$ is $f^{\prime}$. So the question arises, does the oscillation mentioned in the above paragraph occur on the component intervals of this set $O$ ? In what follows, an affirmative answer is furnished to this question. It can be concluded immediately that $f^{\prime}$ has the Darboux property on $O$. In fact $f^{\prime}(I \cap O)=f_{\mathrm{ap}}^{\prime}(I)$ for all open intervals $I \subset I_{0}$.

Presented to the Society, August 21, 1975; received by the editors May 10, 1976.

AMS (MOS) subject classifications (1970). Primary 26A24.

O American Mathematical Society 1977 
In the final section several corollaries of the main results are given.

As a hopefully fitting, but certainly insufficient, tribute, the authors would like to dedicate this paper to Professor Casper Goffman, who for years has been an inspiration and friend to both, and to whom both owe a large debt of gratitude.

2. Conventions, definitions and known results. Throughout this paper all functions are real-valued functions of one variable. The notations $\mu(E)$, $\mathrm{CL}(E)$ and int $E$ will denote the Lebesgue measure, closure and interior of $E$, respectively. The letter $I_{0}$ will designate a fixed interval, that is, a connected subset of the real line. A neighborhood of a point $x$ will be an interval $I$, open relative to $I_{0}$, and containing $x$. A component of an open set $O$ is a maximal subinterval of $O$. The definitions and properties of approximate derivatives and $k$ th Peano derivatives can be found in [1], [6], [4], and [7]. In addition, the following result can be concluded from Theorem 6.1 and the remark at the end of $\$ 8$ of [2].

2.1. THEOREM. If $f_{k}(x)$ exists for all $x$ in $I_{0}$, and if $f_{k}$ is Lebesgue integrable on $[a, b]$, a subinterval of $I_{0}$, then

$$
f_{k-1}(b)-f_{k-1}(a)=\int_{a}^{b} f_{k}(x) d x
$$

3. Preliminary results. This section contains four lemmas which, in addition to Theorem A, will be essential to the proofs of the main results. The first gives rather technical conditions under which a function has an ordinary derivative at a point. In the second and fourth lemmas the reader will see justification for the conditions of the first lemma. The second and third deal with the structure of approximately derivable functions having finite approximate derivatives. The last is yet another version of the so-called Zahorski $\left(\mathrm{M}_{3}\right)$ property (see [9]) for the $k$ th Peano derivative.

3.1. LemMa. Let $f$ be a function, $x$ a point in the domain of $f, \lambda$ a real number, and $K$ a positive number. If for each $\varepsilon>0$ there is an $\delta>0$ such that $0<|y-x|<\delta$ and $y$ in the domain of $f$ imply there are numbers $y_{1}$ and $y_{2}$ with $y_{1}<y<y_{2}$ satisfying:

(1) $\left|f\left(y_{i}\right)-f(x)-\lambda\left(y_{i}-x\right)\right|<\varepsilon\left|y_{i}-x\right|$ for $i=1,2$,

(2) $\left|y_{i}-y\right|<\varepsilon|y-x|$ for $i=1,2$, and

(3) (a) $f(z)+K z$ is increasing on $\left[y_{1}, y_{2}\right]$, or

(b) $f(z)-K z$ is decreasing on $\left[y_{1}, y_{2}\right]$, or

(c) $\left|f\left(y_{i}\right)-f(y)\right|<K\left|y_{i}-y\right|$ for $i=1$ or $i=2$, then $f^{\prime}(x)$ exists and equals $\lambda$.

Proof. Let $\varepsilon>0$ and choose $\delta>0$ as allowed in the hypothesis. Let $y$ be in the domain of $f$ with $0<|y-x|<\delta$ and pick $y_{1}$ and $y_{2}$ satisfying (1)-(3). Suppose first that part (a) of condition (3) holds. Then 


$$
f\left(y_{1}\right)+K y_{1}<f(y)+K y<f\left(y_{2}\right)+K y_{2} .
$$

It follows that

$$
\begin{aligned}
f(y)- & f(x)-\lambda(y-x) \\
& <f\left(y_{2}\right)-f(x)-\lambda\left(y_{2}-x\right)+\lambda\left(y_{2}-y\right)+K\left(y_{2}-y\right) \\
& <\varepsilon\left|y_{2}-x\right|+\lambda\left(y_{2}-y\right)+K\left(y_{2}-y\right) \\
& <\varepsilon\left|y_{2}-y\right|+\varepsilon|y-x|+\lambda\left(y_{2}-y\right)+K\left(y_{2}-y\right) \\
& <\varepsilon^{2}|y-x|+\varepsilon|y-x|+|\lambda| \varepsilon|y-x|+K \varepsilon|y-x| \\
& =(\varepsilon+1+|\lambda|+K) \varepsilon|y-x| .
\end{aligned}
$$

Similarly it can be shown that

$$
-(\varepsilon+1+|\lambda|+K) \varepsilon|y-x|<f(y)-f(x)-\lambda(y-x) .
$$

Consequently,

$$
|f(y)-f(x)-\lambda(y-x)|<(\varepsilon+1+|\lambda|+K) \varepsilon|y-x| .
$$

It can be shown that the same inequality holds if part (b) of condition (3) is assumed. So now suppose part (c) of (3) holds. Then

$$
\begin{aligned}
\mid f(y)-f(x)- & \lambda(y-x)|\leqslant| f(y)-f\left(y_{i}\right) \mid \\
& +\left|f\left(y_{i}\right)-f(x)-\lambda\left(y_{i}-x\right)\right|+\left|\lambda\left(y_{i}-y\right)\right| \\
< & K\left|y_{i}-y\right|+\varepsilon\left|y_{i}-x\right|+|\lambda|\left|y_{i}-y\right| \\
\leqslant & K \varepsilon|y-x|+\varepsilon\left|y_{i}-y\right|+\varepsilon|y-x|+|\lambda| \varepsilon|y-x| \\
< & (K+\varepsilon+1+|\lambda|) \varepsilon|y-x| .
\end{aligned}
$$

So in any of the three cases inequality (1) holds. Now it is easy to see that if for each $\varepsilon>0$ there is a $\delta>0$ such that $y$ in the domain of $f$ and $0<|y-x|<\delta$ implies (1) holds, then $f^{\prime}(x)$ exists and is $\lambda$.

3.2. LeMma. Let $f$ be a function and $x$ a point at which $f$ has an approximate derivative $f_{\text {ap }}^{\prime}(x)=\lambda$. Let $\varepsilon>0$ be given. There is a $\delta>0$ such that $0<\mid y-$ $x \mid<\delta$ implies there are numbers $y_{1}$ and $y_{2}$ with $y_{1}<y<y_{2}$ satisfying

(1) $\left|f\left(y_{i}\right)-f(x)-\lambda\left(y_{i}-x\right)\right|<\varepsilon\left|y_{i}-x\right|$ for $i=1,2$, and

(2) $\left|y_{i}-y\right|<\varepsilon|y-x|$ for $i=1,2$.

Proof. It suffices to consider just $1>\varepsilon>0$. Let

$$
B=\{z:|f(z)-f(x)-\lambda(z-x)|<\varepsilon|z-x|\} .
$$

There is a $\delta>0$ such that, for all closed intervals $I$, containing $x$, of measure less than $\delta(1+\varepsilon)$,

$$
\mu(B \cap I)^{\prime}>\mu(I) /(1+\varepsilon) .
$$

Now let $y$ be fixed with $|x-y|<\delta$. It may be assumed without loss of 
generality that $y>x$. Let $I=[x, y+\varepsilon(y-x)]$. Then

$$
\mu(I)=(1+\varepsilon)(y-x)<\delta(1+\varepsilon) .
$$

Therefore $\mu(B \cap I)>\mu(I) /(1+\varepsilon)=y-x$, and

$$
\mu(B \cap I) \leqslant(1-\varepsilon)(y-x)+\mu(B \cap[y-\varepsilon(y-x), y+\varepsilon(y-x)]) .
$$

Hence

$$
\begin{gathered}
\mu(B \cap[y-\varepsilon(y-x), y+\varepsilon(y-x)])>(y-x)-(1-\varepsilon)(y-x) \\
=\varepsilon(y-x)=\mu([y-\varepsilon(y-x), y+\varepsilon(y-x)]) / 2,
\end{gathered}
$$

which implies the existence of two points $y_{1}<y<y_{2}$ satisfying (1) and (2).

3.3. Lemma. Suppose $f$ is approximately continuous on an interval $I_{0}$. Let $K>0$ be given, and let $A(x)=\{y:|f(y)-f(x)|<K|x-y|\}$. Let $H_{n}=$ $\{x: \mu(A(x) \cap I)>3 \mu(I) / 4$ for all intervals I, containing $x$, with $\mu(I)<$ $1 / n\}$. Then:

(a) If $x$ and $y$ belong to $\mathrm{Cl}\left(H_{n}\right)$ and $|x-y|<1 / n$, then $|f(x)-f(y)|<$ $K|x-y|$.

(b) If $x$ belongs to $\mathrm{Cl}\left(H_{n}\right)$ and $I$ is any interval containing $x$ with $\mu(I)<$ $1 / n$, then $\mu(A(x) \cap I) \geqslant 3 \mu(I) / 4$.

Proof. This is a version of a theorem in [6, p. 499]. An outline of the proof can also be found in [5, pp. 85, 86]. For these reasons a detailed proof is omitted here.

3.4. Lemma. Suppose that for each $x \in I_{0}, f_{k}(x)$ exists and let $j$ be an integer, $0 \leqslant j \leqslant k$. Let $\varepsilon>0$ and let $\left\{I_{n}\right\}$ be a sequence of closed subintervals of $I_{0}$ whose endpoints converge to $x$ as $n$ increases but $x \notin I_{n}$ for each $n$. If for each $n$ and each $y \in I_{n}$,

$$
\begin{aligned}
& \left|f_{j}(y)-f_{j}(x)-f_{j+1}(x)(y-x)-\cdots-f_{k}(x)(y-x)^{k-j} /(k-j) !\right| \\
& \quad \geqslant \varepsilon|y-x|^{k-j} /(k-j) !,
\end{aligned}
$$

then

$$
\lim _{n \rightarrow \infty} \mu\left(I_{n}\right) / \operatorname{dist}\left(I_{n}, x\right)=0,
$$

where

$$
\operatorname{dist}\left(I_{n}, x\right)=\inf \left\{|z-x|: z \in I_{n}\right\} .
$$

The proof of this lemma is essentially like the first part of the proof of Theorem 3, p. 371 of [8] and is therefore omitted. This lemma will be applied in its following equivalent form for the case $j=k-1$.

3.5. Lemma. Suppose that for each $x \in I_{0}, f_{k}(x)$ exists. Then for each $\varepsilon>0$ there is a $\delta>0$ such that if $y \in I_{0}$ and $0<|x-y|<\delta$, then there are 
numbers $y_{1}$ and $y_{2}$ with $y_{1}<y<y_{2}$ satisfying

(1) $\left|f_{k-1}\left(y_{i}\right)-f_{k-1}(x)-\left(y_{i}-x\right) f_{k}(x)\right|<\varepsilon\left|y_{i}-x\right|$ for $i=1,2$, and

(2) $\left|y_{i}-y\right|<\varepsilon|y-x|$ for $i=1,2$.

\section{Main results.}

4.1. THEOREM. Suppose $f$ has a finite approximate derivative, $f_{\mathrm{ap}}^{\prime}(x)$ at each $x$ in $I_{0}$, and let $M \geqslant 0$. If $f_{\text {ap }}^{\prime}$ attains both $M$ and $-M$ on $I_{0}$, then there is a subinterval $I$ of $I_{0}$ on which $f_{\mathrm{ap}}^{\prime}=f^{\prime}$ and $f^{\prime}$ attains both $M$ and $-M$ on $I$.

Proof. Suppose no such interval $I$ exists. Then for each interval $I \subset I_{0}$ on which $f_{\text {ap }}^{\prime}=f^{\prime}, f^{\prime}(y)>-M$ for all $y \in I$ or $f^{\prime}(y)<M$ for all $y \in I$, for otherwise the Darboux property of $f^{\prime}$ would imply that $f^{\prime}$ attains $M$ and $-M$ on $I$. Let

$$
O=\left\{\begin{array}{l}
x \in I_{0}: \text { there is an open interval } I \subset I_{0} \text { such } \\
\text { that } x \in I \text { and } f_{\text {ap }}^{\prime}(y)=f^{\prime}(y) \text { for all } y \in I
\end{array}\right\} .
$$

As mentioned in the introduction $O$ is an open, dense subset of $I_{0}$. Since $f^{\prime}>-M$ or $f^{\prime}<M$ on each component $(a, b)$ of $O$, it follows, from Theorem A, that $f$ has a right-sided derivative at $b$. Thus the set $O$ cannot have two abutting components, and the set $I_{0} \backslash O=P$ is a perfect, nowhere dense set.

Since the function $f_{\text {ap }}^{\prime}$ is Baire $1, P$ contains points at which $f_{\text {ap }}^{\prime}$ is continuous relative to $P$. At any such point $x_{0},\left|f_{\text {ap }}^{\prime}\left(x_{0}\right)\right|<M$. For, suppose that $f_{\text {ap }}^{\prime}\left(x_{0}\right)>M$. (A similar argument holds if $f_{\text {ap }}^{\prime}\left(x_{0}\right)<.-M$.) Then there is an open interval $I$ containing $x_{0}$ for which $f_{\mathrm{ap}}^{\prime}(x)>M$ for $x \in I \cap P$. For any component $(a, b)$ of $O$ with $(a, b) \subset I, a$ is in $I \cap P$, and thus $f_{\text {ap }}^{\prime}(a)>M$. Hence $f_{\text {ap }}^{\prime}(x)>-M$ for $x \in(a, b)$. Combining these two facts, it follows that $f_{\mathrm{ap}}^{\prime}>-M$ on $I$. Therefore $I \subset O$, which contradicts $x_{0} \in P$.

Now by selecting any point $x_{0}$ of $P$ at which $f_{\text {ap }}^{\prime}$ is continuous, relative to $P$, an open interval $(c, d)$ can be determined with $c$ and $d$ in $O, c<x_{0}<d$, and $\left|f_{\text {ap }}^{\prime}(x)\right|<M+1$ on $(c, d) \cap P$. Then for $K=M+1$, the sets $H_{n}$ defined as in Lemma 3.3 have the property that $\cup_{n=1}^{\infty} H_{n} \cap P=P$. The Baire category theorem guarantees the existence of an integer $N$ and an open interval $J$ with $J \cap P \neq \varnothing$ and $J \cap P \subset J \cap \mathrm{CL}\left(H_{N}\right)$. It may be assumed that $\mu(J)<$ $1 / N$ and that the endpoints of $J$ are in $O$. However, as will be shown below, under these conditions $f$ is differentiable on $J$, which contradicts $J \cap P=\varnothing$.

It will be necessary to establish several bounds on the difference quotients $(f(x)-f(y)) /(x-y)$ over the interval $J$. This is done in order to apply Lemma 3.1. It should be remarked that Lemma 3.2 shows conditions (1) and (2) of Lemma 3.1 are satisfied for any point $x$ of $J$. Therefore, only condition (3) need be shown to apply to points of $J$.

Since $\mu(J)<1 / N$ and $J \cap P \subset \operatorname{CL}\left(H_{N}\right) \cap J$, it follows that, for any two 
points $x, y$ of $J \cap P,|f(x)-f(y)| \leqslant(M+1)|x-y|$. Next let $(a, b) \subset J$ be a component of $O$. It is claimed that for all $x$ in $[a, b]$,

$$
|f(x)-f(a)| \leqslant 3(M+1)|x-a|, \quad|f(x)-f(b)|<3(M+1)|x-b| .
$$

It will suffice to prove only the first of these inequalities in the case where $f^{\prime}<M$ on $(a, b)$. The other inequality and the case where $f^{\prime}>-M$ on $(a$, $b$ ) have parallel proofs.

The function $f$ has the mean value property on $[a, b]$; so that for any two points $a \leqslant x \leqslant y \leqslant b, f(y)-f(x)<M(y-x)$. Therefore, it need only be established that $f(x)-f(a) \geqslant(-3)(M+1)(x-a)$ for all $a \leqslant x \leqslant b$. First let $(a+b) / 2 \leqslant x \leqslant b$. Since $a, b \in J \cap P$ it follows that

$$
f(b) \geqslant f(a)-(M+1)(b-a) .
$$

Moreover, by the mean value property

$$
f(x) \geqslant f(b)-M(b-x) .
$$

Thus

$$
f(x)>f(a)-(M+1)(x-a)-(M+1)(b-x)-M(b-x),
$$

and $0 \leqslant b-x \leqslant x-a$. So we have

$$
f(x) \geqslant f(a)-3(M+1)(x-a) .
$$

For $a<x<(a+b) / 2$ let $x_{0}=x+(x-a)<b$. Since $a$ belongs to $\mathrm{CL}\left(H_{N}\right)$ it follows that

$$
\mu\left(A(a) \cap\left[d, x_{0}\right]\right) \geqslant 3 \mu\left(\left[a, x_{0}\right]\right) / 4>x-a .
$$

Therefore, the interval $\left(x, x_{0}\right)$ contains a point $y$ of $A(a)$. Thus

$$
|f(y)-f(a)| \leqslant(M+1)(y-a),
$$

and hence

$$
f(y) \geqslant f(a)-(M+1)(y-a) .
$$

Again by the mean value property

$$
f(x) \geqslant f(y)-M(y-x) .
$$

Finally

$$
x-a \geqslant y-x \geqslant 0 .
$$

Hence

$$
f(x)>f(a)-3(M+1)(x-a) .
$$

It is further shown that for any two points $x<y$ of $J$ which are not in the same component of $O$,

$$
|f(x)-f(y)| \leqslant 3(M+1)|x-y| .
$$

This is clear if $x$ and $y$ both belong to $\operatorname{CL}\left(H_{N}\right) \cap J$. (In this case the factor of 
3 can be replaced by 1.) This leaves three other cases which may occur. However, again it should suffice to indicate the proof for the case where $x$ belongs to $O$ and $y$ belongs to $P$. Let $(a, b)$ be the component of $O$ with $a<x<b \leqslant y$. Then by the above,

$$
|f(x)-f(b)| \leqslant 3(M+1)(b-x) \text {. }
$$

Because $b$ and $y$ belong to $J \cap P$,

$$
|f(b)-f(y)| \leqslant(M+1)(y-b) .
$$

Since $0<y-x=(y-b)+(b-x)$ it follows that

$$
|f(x)-f(y)| \leqslant 3(M+1)|x-y| \text {. }
$$

Therefore if it is assumed that on each component of $O, f^{\prime}>-M$ or $f^{\prime}<M$, then for any point $x_{0}$ of $J$ all the conditions of Lemma 3.1 are satisfied. Hence $f$ is differentiable on $J$, which contradicts $J \cap P \neq \varnothing$, and this completes the proof of Theorem 4.1.

4.2. THEOREM. Suppose $f_{k}(x)$ exists for all $x$ in $I_{0}$ and let $M \geqslant 0$. If $f_{k}$ attains both $M$ and $-M$ on $I_{0}$, then there is a subinterval I of $I_{0}$ on which $f_{k}=f^{(k)}$ and $f^{(k)}$ attains both $M$ and $-M$ on $I$.

Proof. Suppose no such interval exists. Then for each interval $I \subset I_{0}$ on which $f_{k}=f^{(k)}, f^{(k)}(y)>-M$ for all $y \in I$ or $f^{(k)}(y)<M$ for all $y \in I$, again due to the Darboux property. Let

$$
O=\left\{\begin{array}{l}
x \in I_{0}: \text { there is an open interval } I \subset I_{0} \text { such } \\
\text { that } x \in I \text { and } f_{k}(y)=f^{(k)}(y) \text { for all } y \in I
\end{array}\right\} .
$$

Then, as before, $O$ is an open, dense set and, as in the proof of Theorem 4.1, $I_{0} \backslash O=P$ is a perfect set. Further, if $x \in P$ is a point of continuity of $f_{k}$ relative to $P$, then $\left|f_{k}(x)\right| \leqslant M$. So it is possible to select a subinterval $J$ of $I_{0}$, whose endpoints belong to $P$, such that $\left|f_{k}(z)\right| \leqslant M+\frac{1}{2}$ for all $z \in J \cap P$.

For each $n=1,2,3, \ldots$, let

$$
H_{n}=\left\{\begin{array}{l}
x \in J \cap P: \text { for each } y \in I_{0} \text { with } 0<|x-y| \\
<1 / n \text { there are } y_{1}, y_{2} \text { with } y_{1}<y<y_{2} \text { such that } \\
\left|f_{k-1}\left(y_{i}\right)-f_{k-1}(x)\right| \leqslant(M+1)\left|y_{i}-x\right| \text { for } i=1,2 \\
\text { and }\left|y_{i}-y\right| \leqslant|y-x| / 2 \text { for } i=1,2
\end{array}\right\} .
$$

It follows from Lemma 3.5 that $\cup_{n=1}^{\infty} H_{n}=J \cap P$. By the Baire Category Theorem there is an $N$ and an interval $(c, d)$ such that

$$
\varnothing \neq(c, d) \cap(J \cap P) \subset \mathrm{CL}\left(H_{N}\right) .
$$

It may be assumed that $c, d \in J \cap P, d-c<1 / N$ and that $c$ and $d$ are not endpoints of components of $O$. 
The essential fact to establish is that if $(a, b)$ is a component of $O$ with ( $a$, b) $\subset(c, d)$, then

$$
\left|f_{k-1}(b)-f_{k-1}(a)\right| \leqslant 7(M+1)(b-a) .
$$

The proof is presented here for the case where $f^{(k)}(y)<M$ for $y \in(a, b)$. The proof for the other case is similar. Since there is a $z$ in $(a, b)$ with

$$
f_{k-1}(b)-f_{k-1}(a)=f^{(k)}(z)(b-a)<M(b-a),
$$

it suffices to prove

$$
f_{k-1}(b)-f_{k-1}(a)>-7(M+1)(b-a) .
$$

By Lemma 3.5 there is a $\delta>0$ so that $y \in I_{0}$ and $0<|y-a|<\delta$ imply there are $y_{1}, y_{2}$ with $y_{1}<y<y_{2}$ satisfying $\left|f_{k-1}\left(y_{i}\right)-f_{k-1}(a)\right|<\left|y_{i}-a\right|(M$ $+1)$ for $i=1,2$ and $\left|y_{i}-y\right|<|y-a| / 2$ for $i=1,2$. By applying this to a number $y \in(a,(a+b) / 2)$ with $0<|y-a|<\delta$, it follows that there is a $y_{1}$ satisfying

$$
\left|f_{k-1}\left(y_{1}\right)-f_{k-1}(a)\right|<(M+1)\left|y_{1}-a\right|
$$

and $a<y_{1}<(a+b) / 2$. Since $c<a$ and since $H_{N}$ is dense in $(c, d) \cap P$, it follows that there is an $x(a) \in H_{N}$ with $|a-x(a)|<\left|y_{1}-a\right|<(b-a) / 2$. Since $x(a) \in H_{N},(a, b) \subset(c, d)$ and $d-c<1 / N$, there is a $y_{2}<y_{1}$ such that

$$
\left|f_{k-1}\left(y_{2}\right)-f_{k-1}(x(a))\right|<(M+1)\left|y_{2}-x(a)\right|
$$

and $\left|y_{2}-y_{1}\right|<\left|y_{1}-x(a)\right| / 2$. Then

$$
\left|y_{2}-y_{1}\right|<\left|y_{1}-x(a)\right| / 2 \leqslant\left(\left|y_{1}-a\right|+|a-x(a)|\right) / 2 \leqslant\left|y_{1}-a\right| .
$$

So $a<y_{2}<y_{1}<b$. Thus

$$
f_{k-1}\left(y_{1}\right)-f_{k-1}\left(y_{2}\right)<M\left(y_{1}-y_{2}\right) .
$$

Similar to the way $y_{1}$ was chosen it is possible to select $y_{3} \in((a+b) / 2, b)$ such that

$$
\left|f_{k-1}(b)-f_{k-1}\left(y_{3}\right)\right|<(M+1)\left|b-y_{3}\right| .
$$

Also, similar to the way $x(a)$ was produced, one can find $x(b) \in H_{N}$ and a corresponding $y_{4}$ with $y_{3}<y_{4}<b$ such that

$$
\left|f_{k-1}\left(y_{4}\right)-f_{k-1}(x(b))\right|<(M+1)\left|x(b)-y_{4}\right|
$$

and

$$
f_{k-1}\left(y_{4}\right)-f_{k-1}\left(y_{3}\right)<M\left(y_{4}-y_{3}\right) .
$$

Since $x(a) \in(c, d) \cap H_{N}$ and since $b-a<d-c<1 / N$, there is $y_{5}>$ $(a+b) / 2$ such that

$$
\left|f_{k-1}\left(y_{5}\right)-f_{k-1}(x(a))\right|<(M+1)\left|y_{5}-x(a)\right|
$$


and $\left|y_{5}-((a+b) / 2)\right|<|((a+b) / 2)-x(a)| / 2$. Now

$$
\left|y_{5}-((a+b) / 2)\right|<(|((a+b) / 2)-a|+|a-x(a)|) / 2<(b-a) / 2 \text {. }
$$

So $(a+b) / 2<y_{5}<b$. In an analogous fashion there is $y_{6}$ with $a<y_{6}<$ $(a+b) / 2$ such that

$$
\left|f_{k-1}(x(b))-f_{k-1}\left(y_{6}\right)\right|<(M+1)\left|x(b)-y_{6}\right| \text {. }
$$

Since $a<y_{6}<(a+b) / 2<y_{5}<b$,

$$
f_{k-1}\left(y_{5}\right)-f_{k-1}\left(y_{6}\right)<M\left(y_{5}-y_{6}\right) \text {. }
$$

From the displayed formulas of this paragraph it follows that

$$
\begin{aligned}
f_{k-1}(b) & -f_{k-1}(a) \\
= & f_{k-1}(b)-f_{k-1}\left(y_{3}\right)+f_{k-1}\left(y_{3}\right)-f_{k-1}\left(y_{4}\right) \\
& +f_{k-1}\left(y_{4}\right)-f_{k-1}(x(b))+f_{k-1}(x(b)) \\
& -f_{k-1}\left(y_{6}\right)+f_{k-1}\left(y_{6}\right)-f_{k-1}\left(y_{5}\right)+f_{k-1}\left(y_{5}\right) \\
& -f_{k-1}(x(a))+f_{k-1}(x(a))-f_{k-1}\left(y_{2}\right) \\
& +f_{k-1}\left(y_{2}\right)-f_{k-1}\left(y_{1}\right)+f_{k-1}\left(y_{1}\right)-f_{k-1}(a) \\
> & -(M+1)\left|b-y_{3}\right|-M\left|y_{4}-y_{3}\right|-(M+1)\left|x(b)-y_{4}\right| \\
& -M\left|x(b)-y_{6}\right|-M\left|y_{6}-y_{5}\right|-(M+1)\left|y_{5}-x(a)\right| \\
& -(M+1)\left|y_{2}-x(a)\right|-M\left|y_{2}-y_{1}\right|-(M+1)\left|y_{1}-a\right| \\
> & -(M+1)|b-a| / 2-M|b-a| / 2-(M+1)|b-a| / 2 \\
& -(M+1) 3|b-a| / 2-M|b-a|-(M+1) 3|b-a| / 2 \\
& -(M+1)|b-a| / 2-M|b-a| / 2-(M+1)|b-a| / 2 \\
> & -7(M+1)|b-a| .
\end{aligned}
$$

Let $(a, b)$ be a component of $O$ contained in $(c, d)$. Since $f^{(k)}$ is bounded above or below on $[a, b], f^{(k)}$ is Lebesgue integrable on $(a, b)$ and $f_{k-1}(b)$ $f_{k-1}(a)=\int_{a}^{b} f^{(k)}(t) d t$. Since

$$
\left|\int_{a}^{b} f^{(k)}(t) d t\right|=\left|f_{k-1}(b)-f_{k-1}(a)\right|<7(M+1)|b-a|,
$$

and since $f^{(k)}$ is bounded above or below on $[a, b]$, it follows that

$$
\int_{a}^{b}\left|f^{(k)}(t)\right| d t \leqslant\left|\int_{a}^{b} f^{(k)}(t) d t\right|+M(b-a)<8(M+1)(b-a) .
$$

Since $\left|f_{k}\right|<M+\frac{1}{2}$ on $(c, d) \cap P \subset J \cap P$, it follows that $f_{k}$ is Lebesgue integrable on $(c, d)$. According to Theorem 2.1 for any $c \leqslant x<y<d$,

$$
f_{k-1}(y)-f_{k-1}(x)=\int_{x}^{y} f_{k}(t) d t
$$


Next it is shown that if $(a, b)$ is a component of $O \cap(c, d)$ and if $y \in(a$, b), then

$$
\left|f_{k-1}(y)-f_{k-1}(a)\right| \leqslant 15(M+1)(y-a)
$$

and

$$
\left|f_{k-1}(b)-f_{k-1}(y)\right|<15(M+1)(b-y) .
$$

First let $\left\{x_{m}\right\}$ be a sequence from $H_{N}$ converging to $a$. Then for each $m$ there are $y_{1, m}<y<y_{2, m}$ such that

$$
\left|f_{k-1}\left(y_{i, m}\right)-f_{k-1}\left(x_{m}\right)\right|<(M+1)\left|y_{i, m}-x_{m}\right| \text { for } i=1,2
$$

and $\left|y_{i, m}-y\right|<\left|y-x_{m}\right| / 2$ for $i=1,2$. Clearly each of the sequences $\left\{y_{i, m}\right\}, i=1,2$, is bounded. So we may assume that each converges, say to $y_{i}$. Then $\left|y_{i}-y\right|<|y-a| / 2$. Since $f_{k-1}$ is an indefinite integral, it is continuous. Thus

$$
\left|f_{k-1}\left(y_{i}\right)-f_{k-1}(a)\right|<(M+1)\left|y_{i}-a\right| \text { for } i=1,2 .
$$

Again assume that $f^{(k)}(z)<M$ for $z \in(a, b)$. The argument for $f^{(k)}(z)>-$ $M$, being similar, is omitted. Then $f_{k-1}(y)-f_{k-1}(a)<M(y-a)$; so it suffices to prove that $f_{k-1}(y)-f_{k-1}(a)>-15(M+1)(y-a)$. First suppose that $a<y<(a+b) / 2$. Since $\left|y_{2}-y\right|<|y-a| / 2<|b-a| / 2$, $y_{2} \in(a, b)$. Thus $f_{k-1}\left(y_{2}\right)-f_{k-1}(y)<M\left(y_{2}-y\right)$. Therefore

$$
\begin{aligned}
f_{k-1}(y) & -f_{k-1}(a)=f_{k-1}(y)-f_{k-1}\left(y_{2}\right)+f_{k-1}\left(y_{2}\right)-f_{k-1}(a) \\
& >-M\left(y_{2}-y\right)-(M+1)\left(y_{2}-a\right) \\
& >-M|y-a| / 2-(M+1)\left(y_{2}-y+y-a\right) \\
& >-M|y-a| / 2-(M+1) 3|y-a| / 2>-2(M+1)|y-a| .
\end{aligned}
$$

Second, suppose $(a+b) / 2<y<b$. Then

$$
\begin{aligned}
f_{k-1}(y) & -f_{k-1}(a)=f_{k-1}(y)-f_{k-1}(b)+f_{k-1}(b)-f_{k-1}(a) \\
& >-M(b-y)-7(M+1)(b-a) \\
& >-M|b-a| / 2-14(M+1)|b-a| / 2 \\
& >-15(M+1)|b-a| / 2>-15(M+1)|y-a| .
\end{aligned}
$$

The proof of the inequality involving $b$ is similar to the one given for $a$.

Now it is established that if $x \in(c, d) \backslash O$ and $y \in(c, d)$, then

$$
\left|f_{k-1}(y)-f_{k-1}(x)\right|<15(M+1)|y-x| .
$$

If $y \in(c, d) \backslash O$, then denote by $L$ the closed interval with endpoints $x$ and $y$. Hence 


$$
\begin{aligned}
\left|f_{k-1}(y)-f_{k-1}(x)\right| & \leqslant \int_{L}\left|f_{k}(t)\right| d t=\int_{L \cap o}\left|f_{k}(t)\right| d t+\int_{L \backslash O}\left|f_{k}(t)\right| d t \\
& <8(M+1) \mu(L \cap O)+(M+1) \mu(L \backslash O) \\
& \leqslant 8(M+1)|y-x| .
\end{aligned}
$$

If $y \in(c, d) \cap O$, then let $a$ be that endpoint of the component of $O$ containing $y$ which lies between $x$ and $y$. Then

$$
\begin{aligned}
& \left|f_{k-1}(y)-f_{k-1}(x)\right| \leqslant\left|f_{k-1}(y)-f_{k-1}(a)\right|+\left|f_{k-1}(a)-f_{k-1}(x)\right| \\
& \quad \leqslant 15(M+1)|y-a|+8(M+1)|a-x| \leqslant 15(M+1)|y-x| .
\end{aligned}
$$

The concluding step is to verify that for each $x \in(c, d) \backslash O, f_{k-1}^{\prime}(x)=$ $f_{k}(x)$. It will then follow that $(c, d) \subset O$ contrary to $\varnothing \neq(c, d) \cap P$. Let $x \in(c, d) \backslash O$ and let $\varepsilon>0$. Then by Lemma 3.5 there is a $\delta>0$ such that $0<|x-y|<\delta$ implies there are $y_{1}<y<y_{2}$ such that

(1) $\left|f_{k-1}\left(y_{i}\right)-f_{k-1}(x)-\left(y_{i}-x\right) f_{k}(x)\right|<\varepsilon\left|y_{i}-x\right|$ for $i=1,2$, and

(2) $\left|y_{i}-x\right|<\varepsilon|y-x|$ for $i=1,2$.

Also insist that $\delta$ is so small that not only does $y \in(c, d)$ but $y_{1}, y_{2} \in(c, d)$ as well. Then there are three cases:

(a) $\left[y_{1}, y_{2}\right]$ is a subinterval of an interval where $f^{(k)}>-M$, or

(b) $\left[y_{1}, y_{2}\right]$ is a subinterval of an interval where $f^{(k)}<M$, or

(c) $\left[y_{1}, y_{2}\right] \cap P \neq \varnothing$.

In case (a) $f_{k-1}(z)+M z$ is increasing on $\left[y_{1}, y_{2}\right]$. In case (b) $f_{k-1}(z)-M z$ is decreasing on $\left[y_{1}, y_{2}\right]$. In case (c) let $x^{\prime} \in\left[y_{1}, y_{2}\right] \cap P$. If $y_{1}<x^{\prime} \leqslant y$, then

$$
\left|f_{k-1}\left(x^{\prime}\right)-f_{k-1}\left(y_{1}\right)\right| \leqslant 15(M+1)\left|x^{\prime}-y_{1}\right|
$$

and

$$
\left|f_{k-1}(y)-f_{k-1}\left(x^{\prime}\right)\right| \leqslant 15(M+1)\left|y-x^{\prime}\right| .
$$

So since $x^{\prime}$ is between $y_{1}$ and $y$,

$$
\left|f_{k-1}(y)-f_{k-1}\left(y_{1}\right)\right| \leqslant 15(M+1)\left|y-y_{1}\right| \text {. }
$$

If $y \leqslant x^{\prime}<y_{2}$ it can be shown in a similar fashion that

$$
\left|f_{k-1}\left(y_{2}\right)-f_{k-1}(y)\right| \leqslant 15(M+1)\left|y-y_{2}\right| \text {. }
$$

So it follows that the conditions of Lemma 3.1 are satisfied with $f$ replaced by $f_{k-1}, \lambda$ replaced by $f_{k}(x)$ and $K=15(M+1)$. Hence $f_{k-1}^{\prime}(x)=f_{k}(x)$.

5. In this last section applications of Theorems 4.1 and 4.2 are given. To shorten the exposition these theorems are stated for functions which possess a finite approximate derivative at every point of an interval $I_{0}$. However, in each case a corresponding result for the $k$ th Peano derivative is also valid and with a proof constructed in the same fashion. In compliance with standard notation, for any function $\Delta_{f}$ will denote $\left\{x: f^{\prime}(x)\right.$ exists $\}$. 
5.1 THEOREM. Let $f$ have a finite approximate derivative $f_{\mathrm{ap}}^{\prime}(x)$ for each $x$ in $I_{0}$ and let $\alpha$ be a real number. If $\left\{x: f_{\mathrm{ap}}^{\prime}(x)=\alpha\right\} \neq \varnothing$, then there is an $x \in$ int $\Delta_{f}$ such that $f^{\prime}(x)=\alpha$.

Proof. It may be assumed that $\Delta_{f} \neq I_{0}$, for otherwise the conclusion is obvious. Let $M$ be any number with $M>|\alpha|$. Theorem 4.1 guarantees the existence of a component $(a, b)$ of int $\Delta_{f}$ on which $f^{\prime}$ takes on the values $M$ and $-M$. Since $f^{\prime}$ has the Darboux property on $(a, b), f^{\prime}$ also attains $\alpha$ on $(a$, b).

5.2 COROllaRY. Let $f$ have a finite approximate derivative $f_{\text {ap }}^{\prime}(x)$ for each $x$ in $I_{0}$. If $\{x: f(x)=0\}$ is dense in $I_{0}$, then $f$ is identically zero on $I_{0}$.

5.3 COROLlaRY. Let $f$ and $g$ have finite approximate derivatives $f_{\mathrm{ap}}^{\prime}(x)$ and $g_{\text {ap }}^{\prime}(x)$, respectively, for each $x$ in $I_{0}$. If $\{x: f(x)=g(x)\}$ is dense in $I_{0}$, then $f=g$ on $I_{0}$.

5.4 COROllary. Let $f$ have a finite approximate derivative $f_{\mathrm{ap}}^{\prime}(x)$ and $g$ a finite derivative $g^{\prime}(x)$ for each $x$ in $I_{0}$. If $f^{\prime}=g^{\prime}$ on int $\Delta_{f}$, then $f^{\prime}=g^{\prime}$ on $I_{0}$.

Proof. Let $h=f-g$. Then $h$ has a finite approximate derivative on $I_{0}$ and $\Delta_{h}=\Delta_{f}$. Moreover, $h^{\prime}=0$ on $\Delta_{h}$. Theorem 5.1 guarantees that $h_{\text {ap }}^{\prime}=0$ on $I_{0}$ and the conclusion follows.

Theorem 4.1 also leads easily to the following very general theorem about the monotonicity of approximately derivable functions.

5.5 THEOREM. Let $P$ be a property of functions so that any function which is differentiable and possesses $P$ on an interval $I$ is monotone on I. If $f$ has a finite approximate derivative $f_{\mathrm{ap}}^{\prime}(x)$ at each $x$ in $I_{0}$ and if $f$ has property $P$ on $I_{0}$, then $f$ is monotone on $I_{0}$.

Proof. It suffices to show that $f_{\text {ap }}^{\prime}$ is unisigned on $I_{0}$ (see [1]). Suppose not. It follows from Theorem 4.1 that there is a subinterval $I$ of $I_{0}$ on which $f_{\text {ap }}^{\prime}=f^{\prime}$ and $f^{\prime}$ attains both positive and negative values. Then $f$ is not monotone on $I$ contrary to assumption.

Before proceeding to the last application, it must be pointed out that Lemma 3.3 implies the following known result [6].

Let $f$ have a finite approximate derivative on $I_{0}$ and let $C$ be a nonempty closed subset of $I_{0}$. Then there is an open interval $J$, with $J \cap C \neq \varnothing$, such that $f$ is continuous relative to $C$ on $J \cap C$.

The corresponding result for the $k$ th Peano derivative can be obtained as follows. First observe that if $f_{k}(x)$ exists for each $x$ in $I_{0}$ then $f_{k}$ is $C_{k} P$ integrable on $I_{0}$. Then note that $f_{k-1}$ is an indefinite integral of $f_{k}$. Finally use that an indefinite $C_{k} P$-integral is continuous on a portion of a given closed set (see [7, p. 320, Lemma 5] for references to all of these facts). The result 
can also be proved directly as is indicated below. For a function $f$ defined on $I_{0}$ and $x$ in $I_{0}$ define $\tilde{\Delta}_{k}(f ; x, h)$ inductively by

$$
\begin{aligned}
& \tilde{\Delta}_{1}(f ; x, h)=f(x+h)-f(x), \\
& \tilde{\Delta}_{k}(f ; x, h)=\tilde{\Delta}_{k-1}(f ; x, 2 h)-2^{k-1} \tilde{\Delta}_{k-1}(f ; x, h) .
\end{aligned}
$$

Then

(1) $\tilde{\Delta}_{k-1}(f ; x, h) /(2 h)^{k-1}-\tilde{\Delta}_{k-1}(f ; x, h) / h^{k-1}=h \tilde{\Delta}_{k}(f ; x, h) / 2^{k-1}$

and there is a number $\lambda_{k}$ depending only on $k$ (and not on $f$ or $x$ ) such that if $f_{k}(x)$ exists, then

$$
\lim _{h \rightarrow 0} \lambda_{k} \tilde{\Delta}_{k}(f ; x, h) / h^{k}=f_{k}(x)
$$

(see $\left[3\right.$, pp. 9, 10]).Assuming that $f_{k}(x)$ exists for all $x$ in $I_{0}$, define for $n=1$, $2,3, \ldots$,

$$
F_{n}(x)=\lambda_{k-1} \tilde{\Delta}_{k-1}\left(f ; x, 2^{-n}\right) /\left(2^{-n}\right)^{k-1} .
$$

By (2) $\lim _{n \rightarrow \infty} F_{n}(x)=f_{k-1}(x)$, and since $f$ is continuous, it is easy to see that each $F_{n}$ is continuous. Moreover, by (1) for each $n$,

$$
\begin{aligned}
F_{n}(x)-F_{n+1}(x) & =\lambda_{k-1} \tilde{\Delta}_{k}\left(f ; x, 2^{-n-1}\right) 2^{k-1} \\
& =\left(\lambda_{k-1} / \lambda_{k} 2^{k-1}\right) 2^{-n-1} \lambda_{k} \tilde{\Delta}_{k}\left(f ; x, 2^{-n-1}\right) .
\end{aligned}
$$

For each pair $N, n$ of positive integers, let

$$
E_{N, n}=\left\{x \in I_{0}:\left|F_{n}(x)-F_{n+1}(x)\right|<N 2^{-n-1}\right\}
$$

and

$$
E_{N}=\bigcap_{n=1}^{\infty} E_{N, n}
$$

Since each $F_{n}$ is continuous, each $E_{N, n}$ is closed and, thus, so is $E_{N}$. Moreover, $\left\{F_{n}\right\}$ converges uniformly to $f_{k-1}$ on $E_{N}$. From (2) it follows that

$$
I_{0}=\bigcup_{N=1}^{\infty} E_{N}
$$

It is easy to prove that these conditions on the sequences $\left\{F_{n}\right\}$ and $\left\{E_{N}\right\}$ are equivalent to the statement that for each nonempty closed set $C \subset I_{0}$ there is an open interval $J$ with $J \cap C \neq \varnothing$ and such that $f_{k-1}$ is continuous on $J \cap C$ relative to $C$. 
The above results are used as a basis to prove the following theorem and its analogue for Peano derivatives concerning relative maxima. A function $f$ defined on $I_{0}$ has a relative maximum at $x \in I_{0}$ if there is an interval $(a, b)$ with $x \in(a, b)$ such that for each $y \in I_{0} \cap(a, b), f(y) \leqslant f(x)$.

5.6. THEOREM. Let $f$ have a finite approximate derivative on a closed interval $I_{0}$. Then $f$ has a relative maximum.

Proof. If $\Delta_{f}=I_{0}$ then $f$ is continuous and there is nothing to show. Therefore, only the case where $I_{0} \neq$ int $\Delta_{f}$ must be considered. Assume in this case that on no component $(c, d)$ of int $\Delta_{f}$ does $f$ have a relative maximum. Since $f$ is continuous on $(c, d)$ it is then elementary that on $(c, d)$ either

(1) $f$ is increasing on $(c, d)$; or

(2) $f$ is decreasing on $(c, d)$; or

(3) there is a point $x_{0}$ with $c<x_{0}<d$ for which $f$ is decreasing on (c, $x_{0}$ ] and increasing on $\left[x_{0}, d\right)$.

Since $f$ has the Darboux property on $[c, d]$, each of the three cases actually holds on $[c, d]$. These three cases also imply that no two intervals of int $\Delta_{f}$ can be abutting. Hence, $I_{0} \backslash$ int $\Delta_{f}=C$ is a perfect nowhere dense set. Further, from Theorem 5.5 with $P$ being " $f$ ' is unisigned on each component of int $\Delta_{f}$ ", it follows that the endpoints of those components of int $\Delta_{f}$ which satisfy case (3) are dense in $C$. For suppose there is a point $x_{0}$ of $C$ and a $\delta>0$ such that for all components of int $\Delta_{f}$ inside $\left(x_{0}-\delta, x_{0}+\delta\right)$ only cases (1) and (2) hold. Then $f$ is monotone on $\left(x_{0}-\delta, x_{0}+\delta\right)$, and $\left(x_{0}-\delta\right.$, $\left.x_{0}+\delta\right)$ is contained in int $\Delta_{f}$. Now the remark preceding this theorem guarantees the existence of an open interval $(r, s)$, with $(r, s) \cap C \neq \varnothing$ and $f$ continuous relative to $C$ on $(r, s)$. The interval $(r, s)$ can be chosen so that $r$ and $s$ belong to components of int $\Delta_{f}$ where case (3) holds. In fact, let $\left(c_{1}, d_{1}\right)$ be the component containing $r$ and $\left(c_{2}, d_{2}\right)$ the component containing $s$. It can be assumed that the function $f$ takes on values greater than $f(r)$ on $\left(r, d_{1}\right)$ and values greater than $f(s)$ on $\left(c_{2}, s\right)$. Now $f$ is continuous relative to $C$ on $[r$, $s]$, and if $f$ satisfies cases (1), (2), and (3) on $[r, s] \backslash C$ it can be easily seen that $f$ is upper semicontinuous on $[r, s]$. Hence $f$ has an absolute maximum on $[r$, $s]$. This absolute maximum is not attained at $r$ or $s$. Hence $f$ has a relative maximum on $I_{0}$.

\section{BIBLIOGRAPHY}

1. C. Goffman and C. J. Neugebauer, On approximate derivatives, Proc. Amer. Math. Soc. 11 (1960), 962-966.

2. R. D. James, Generalized nth primitives, Trans. Amer. Math. Soc. 76 (1954), 149-176.

3. J. Marcinkiewicz and A. Zygmund, On the differentiability of functions and summability of trigonometrical series, Fund. Math. 26 (1936), 1-43.

4. H. W. Oliver, The exact Peano derivative, Trans. Amer. Math. Soc. 76 (1954), 444-456. 
5. R. J. O'Malley, A density property and applications, Trans. Amer. Math. Soc. 199 (1974), 75-87.

6. G. Tolstoff, Sur le dérivée approximative exact, Mat. Sb. 4 (1938), 499-504.

7. S. Verblunsky, On the Peano derivatives, Proc. London. Math. Soc. (3) 22 (1971), 313-324.

8. C. E. Weil, On properties of derivatives, Trans. Amer. Math. Soc. 114 (1965), 363-376.

9. Z. Zahorski, Sur la première dérivee, Trans. Amer. Math. Soc. 69 (1950), 1-54.

Department of Mathematics, Universtry of Wisconsin, MilwauKee, Wisconsin 53201

Department of Mathematics, Michigan State University, East lansing, Michigan 48824 